\title{
Front Matter: Volume 10918
}

, "Front Matter: Volume 10918," Proc. SPIE 10918, Gallium Nitride Materials and Devices XIV, 1091801 (7 May 2019); doi: 10.1117/12.2531211

SPIE. Event: SPIE OPTO, 2019, San Francisco, California, United States 


\title{
PROCEEDINGS OF SPIE
}

\section{Gallium Nitride Materials and Devices XIV}

\author{
Hiroshi Fujioka \\ Hadis Morkoç \\ Ulrich T. Schwarz \\ Editors
}

4-7 February 2019

San Francisco, California, United States

Sponsored and Published by

SPIE 
The papers in this volume were part of the technical conference cited on the cover and title page. Papers were selected and subject to review by the editors and conference program committee. Some conference presentations may not be available for publication. Additional papers and presentation recordings may be available online in the SPIE Digital Library at SPIEDigitalLibrary.org.

The papers reflect the work and thoughts of the authors and are published herein as submitted. The publisher is not responsible for the validity of the information or for any outcomes resulting from reliance thereon.

Please use the following format to cite material from these proceedings:

Author(s), "Title of Paper," in Gallium Nitride Materials and Devices XIV, edited by Hiroshi Fujioka, Hadis Morkoç, Ulrich T. Schwarz, Proceedings of SPIE Vol. 10918 (SPIE, Bellingham, WA, 2019) Sevendigit Article CID Number.

ISSN: 0277-786X

ISSN: 1996-756X (electronic)

ISBN: 9781510624788

ISBN: 9781510624795 (electronic)

Published by

SPIE

P.O. Box 10, Bellingham, Washington 98227-0010 USA

Telephone +1 3606763290 (Pacific Time) · Fax +1 3606471445

SPIE.org

Copyright (C) 2019, Society of Photo-Optical Instrumentation Engineers.

Copying of material in this book for internal or personal use, or for the internal or personal use of specific clients, beyond the fair use provisions granted by the U.S. Copyright Law is authorized by SPIE subject to payment of copying fees. The Transactional Reporting Service base fee for this volume is $\$ 18.00$ per article (or portion thereof), which should be paid directly to the Copyright Clearance Center (CCC), 222 Rosewood Drive, Danvers, MA 01923. Payment may also be made electronically through CCC Online at copyright.com. Other copying for republication, resale, advertising or promotion, or any form of systematic or multiple reproduction of any material in this book is prohibited except with permission in writing from the publisher. The CCC fee code is 0277$786 \mathrm{X} / 19 / \$ 18.00$.

Printed in the United States of America by Curran Associates, Inc., under license from SPIE.

Publication of record for individual papers is online in the SPIE Digital Library.

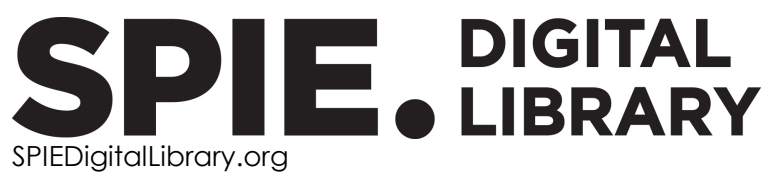

Paper Numbering: Proceedings of SPIE follow an e-First publication model. A unique citation identifier (CID) number is assigned to each article at the time of publication. Utilization of CIDs allows articles to be fully citable as soon as they are published online, and connects the same identifier to all online and print versions of the publication. SPIE uses a seven-digit CID article numbering system structured as follows:

- The first five digits correspond to the SPIE volume number.

- The last two digits indicate publication order within the volume using a Base 36 numbering system employing both numerals and letters. These two-number sets start with $00,01,02,03,04$, 05, 06, 07, 08, 09, 0A, OB ... 0Z, followed by 10-1Z, 20-2Z, etc. The CID Number appears on each page of the manuscript. 


\title{
Contents
}

\author{
vii Authors \\ ix Conference Committee
}

\section{GROWTH II}

10918 OA Enhanced optical and structural properties of MBE-grown AIGaN nanowires on Si substrate by H-ion implantation and UV ozone treatment [10918-9]

\section{MATERIAL CHARACTERIZATION I}

10918 OE III-nitride vertical resonant cavity light-emitting diodes with hybrid air-gap/AIGaN-dielectric distributed Bragg reflectors [10918-13]

$1091801 \quad$ Optically pumped room temperature low threshold deep UV lasers grown on native AIN substrates [10918-17]

\section{MATERIAL CHARACTERIZATION II}

$109180 \mathrm{M}$ Green InGaN/GaN based LEDs: high luminance and blue shift [10918-21]

1091800 Investigation of the device degradation for commercial light-emitting diodes (LEDs) using spatially and time-resolved electro- and photoluminescence [10918-23]

$109180 Q \quad$ Analysis on light extraction property of AIGaN-based flip-chip ultraviolet light-emitting diodes by the use of self-assembled $\mathrm{SiO}_{2}$ microsphere array [10918-25]

\section{NANOSTRUCTURES}

$109180 Z \quad$ New physics in GaN resonant tunneling diodes (Invited Paper) [10918-34]

1091814 Demonstration of uniform and reliable GaN p-i-p-i-n separate-absorption and multiplication ultraviolet avalanche photodiode arrays with large detection area [10918-39]

\section{ELECTRON DEVICES}

1091816 Vertical power devices enabled by bulk GaN substrates (Invited Paper) [10918-41] 
1091817 Degradation physics of GaN-based lateral and vertical devices (Invited Paper) [10918-42]

1091819 Simple ohmic contact formation in HEMT structures: application to AIGaN/GaN [10918-44]

$109181 \mathrm{~A} \quad$ Normally-off p-GaN gate InAIN/GaN HEMTs grown on silicon substrates [10918-45]

10918 1B Electrical and structural characteristics of aged RF GaN HEMTs and irradiated high-power GaN HEMTs with protons and heavy ions [10918-46]

\section{LASER DIODES}

10918 1D High-efficiency blue and green laser diodes for laser displays (Invited Paper) [10918-48]

$109181 \mathrm{~F} \quad$ Influence of sandwiched GaN/AIGaN/GaN lower quantum barrier on crystallinity and luminescence of an asymmetric GaN-based high-power laser diode [10918-50]

VCSEL I

$109181 \mathrm{H} \quad$ Room temperature continuous wave lasing of GaN-based green vertical-cavity surfaceemitting lasers (Invited Paper) [10918-52]

VCSEL II

$109181 \mathrm{~J} \quad$ Recent progress in GaN-based vertical-cavity surface-emitting lasers with lateral optical confinement due to an incorporated curved mirror (Invited Paper) [10918-54]

$109181 \mathrm{~L} \quad$ Influence of Al ion implantation on electrical and optical properties in nitride TJ VCSEL [10918-56]

$109181 \mathrm{M}$ Development of nanopore-based near ultraviolet vertical-cavity surface emitting lasers [10918-57]

\section{MICRO LEDs I}

1091810 GaN monolithic integration for lighting and display (Invited Paper) [10918-59]

$109181 Q \quad$ Advanced solutions for high-performance GaN MicroLED displays (Invited Paper) [10918-61]

iv 
MICRO LEDs II

10918 1R Growth of monolithic full-color light-emitting diode and its applications (Invited Paper) [10918-62]

UV LEDS

$109181 \mathrm{X}$ Functional integrity and stable high-temperature operation of planarized ultraviolet-A Alx $\mathrm{Ga}_{1-\mathrm{x}}$ $\mathrm{N} / \mathrm{Al}_{\mathrm{y}} \mathrm{Ga}_{1-\mathrm{y}} \mathrm{N}$ multiple-quantum-disk nanowire LEDs with charge-conduction promoting interlayer [10918-68]

LEDs

1091822 Suppression of indium clustering and quantum confined stark effect of InGaN LED on silicon (111) [10918-73] 
Proc. of SPIE Vol. 10918 1091801-6

Downloaded From: https://www.spiedigitallibrary.org/conference-proceedings-of-spie on 26 Apr 2023 Terms of Use: https://www.spiedigitallibrary.org/terms-of-use 


\section{Authors}

Numbers in the index correspond to the last two digits of the seven-digit citation identifier (CID) article numbering system used in Proceedings of SPIE. The first five digits reflect the volume number. Base 36 numbering is employed for the last two digits and indicates the order of articles within the volume. Numbers start with 00, 01, 02, 03, 04, 05, 06, 07, 08, 09, OA, OB...0Z, followed by 10-1Z, 20-2Z, etc.

Alatawi, Abdullah A., $1 \mathrm{X}$ Albadri, Abdulrahman M., $1 \mathrm{X}$

Albrecht, John D., Ol

Alfaraj, Nasir, $1 \mathrm{X}$

Alhamoud, Abdullah A., $1 X$

Alyamani, Ahmed Y., $1 \mathrm{X}$

Anderson, Travis J., 16

Ayon, Arturo A., 19

Babu, Sachidananda, 14

Bakhtiary-Noodeh, Marzieh, 14

Barbato, Alessandro, 17

Barbato, Marco, 17

Bayram, Can, 22

Bédoin, Alexis, $1 Q$

Bernard, Jeannet, $1 Q$

Biswas, Mahitosh, OA

Bonsall, Jeremy, 1B

Borga, Matteo, 17

Bozok, Berkay, 1A

Brodie, Miles, 1B

Butun, Bayram, 1A

Canato, Eleonora, 17

Caplet, Stéphane, 1Q

Cetnar, John S., 19

Chakrabarti, Subhananda, OA

Chen, J. C., IR

Chen, Songtao, $1 \mathrm{M}$

Cheung, Y. F., 10

Chiocchetta, Francesca, 17

Cho, Sang June, 01

Choi, H. W., 10

Daami, Anis, OM

Dalmau, Rafael, ol

Das, Debabrata, OA

De Santi, Carlo, 17

Detchprohm, Theeradetch, OE, 14

Dhar, Nibir K., 14

Dupré, Ludovic, OM

Dupuis, Russell D., OE, 14

ElAfandy, Rami T., IM

El-Ghoroury, Hussein S., IR

Encomendero, Jimy, $0 Z$

Fabris, Elena, 17

Feigelson, Boris N., 16

Foran, Brendan, 1B

Fu, W. Y., 10

Gallagher, James C., 16

Ghasempour, Askari, 00

Ghuman, P. Parminder, 14
Gulseren, Melisa Ekin, 1A

Haas, Helge, 1Q

Hamaguchi, Tatsushi, $1 \mathrm{~J}$

Han, Jung, $1 \mathrm{M}$

Hartensveld, Matthew, $0 Q$

Hayashi, Kentaro, $1 \mathrm{~J}$

Heller, Eric, 19

Henry, Franck, OM

Hirao, Tsuyoshi, 1D

Hite, Jennifer K., 16

Hobart, Karl D., 16

Huang, Rui, $1 \mathrm{~F}$

Ito, Masamichi, $1 \mathrm{~J}$

Jacobs, Alan G., 16

Janjua, Bilal, $1 \mathrm{X}$

Jena, Debdeep, $0 Z$

Jeong, Hoon, $\mathrm{OE}, 14$

$\mathrm{Ji}$, Mi-Hee, 14

Jyokawa, Tatsuro, $1 \mathrm{~J}$

Kalapala, Akhil R. K., Ol

Kayal, Omer Ahmet, $1 \mathrm{~A}$

Kobayashi, Noriko, $1 \mathrm{~J}$

Koda, Rintaro, $1 \mathrm{~J}$

Koehler, Andrew D., 16

Kozuru, Kazuma, 1D

Kub, Francis J., 16

Kumar, Raman, OA

Kumar, Ravinder, OA

Kurt, Gokhan, 1A

Lan, Tian, IF

Le Calvez, Stéphanie, OM

Lewis, Jay, 14

Li, K. H., 10

Li, Ying, IF

Licitra, Christophe, OM

Lingley, Zachary, 1B

Liu, Cheng, OQ

Liu, Dong, $\mathrm{Ol}$

Liu, J. P., $1 \mathrm{H}$

Liu, Richard, 22

Long, $\mathrm{H}$., $1 \mathrm{H}$

Look, David C., 19

Luna, Lunet E., 16

Ma, Zhenqiang, 0 I

Masin, Fabrizio, 17

Masui, Shingo, 1D

Mato, Tatsuya, $1 \mathrm{~J}$

McCormick, Callan, 22

Mehta, Karan, OE 
Mei, Y., $1 \mathrm{H}$

Melanson, Bryan, $\mathrm{OQ}$

Meneghesso, Gaudenzio, 17

Meneghini, Matteo, 17

$\mathrm{Mi}$, Zetian, OA

Moody, Baxter, ol

Nagahama, Shin-ichi, 1D

Nagao, Yoji, 1D

Nakajima, Hiroshi, 1 J

Nakajima, Yoshitake, IR

Nakatsu, Yoshitaka, ID

Nardo, Arianna, 17

$\mathrm{Ndi}$, Francis, 00

$\mathrm{Ng}$, Tien Khee, $1 \mathrm{X}$

Nurmikko, Arto, $1 \mathrm{M}$

Ohara, Maho, $1 \mathrm{~J}$

Okahisa, Eiichiro, 1D

Olivier, François, OM

Ooi, Boon S., IX

Ooi, Yu Kee, OQ

Ozbay, Ekmel, 1A

Ozturk, Mustafa, 1 A

Pan, Xinhua, 00

Panda, Debiprasad, OA

Park, Jeongpil, 0 I

Park, Young-Jae, OE

Priante, Davide, $1 \mathrm{X}$

Price, Alain, 00

Rahimi, Nassim, 00

Rampazzo, Fabiana, 17

Ruzzarin, Maria, 17

Sarzała, R. P., $1 \mathrm{~L}$

Shen, Shyh-Chiang, OE, 14

Sin, Yongkun, 1B

Sitzman, Scott, 1B

Sood, Ashok K., 14

Śpiewak, P., 1L

Tajalli, Alaleh, 17

Tanaka, Masayuki, $1 \mathrm{~J}$

Templier, François, OM, 1Q

Tsou, Chuan-Wei, OE

Upadhyay, Sourabh, OA

Ural, Sertac, IA

Vazquez-Colon, Clarissa D., 19

Veksler, Dmitry, 1B

Wang, Congcong, IF

Wang, Jialin, OE

Wang, Zhiyong, $1 \mathrm{~F}$

Wasiak, M., 1L

Watanabe, Hideki, $1 \mathrm{~J}$

Xing, Huili Grace, $\mathrm{OZ}$

$X u, R . B ., 1 H$

Yan, Anru, $1 \mathrm{~F}$

Yanamoto, Tomoya, 1D

Yanashima, Katsunori, $1 \mathrm{~J}$

Yeh, Milton, 1R

Ying, L. Y., $1 \mathrm{H}$

Yoder, P. Douglas, OE

Zanoni, Enrico, 17

Zhang, B. P., $1 \mathrm{H}$
Zhang, Cheng, $1 \mathrm{M}$

Zhang, Jerry, $1 M$

Zhang, Jing, $O Q$

Zhao, Deyin, Ol

Zhao, Songrui, OA

Zheng, Z. W., $1 \mathrm{H}$

Zhou, Guangzheng, $1 \mathrm{~F}$

Zhou, Weidong, 01 


\section{Conference Committee}

Symposium Chairs

Connie J. Chang-Hasnain, University of California, Berkeley (United States)

Graham T. Reed, Optoelectronics Research Center (United Kingdom)

Symposium Co-chairs

Sailing He, KTH Royal Institute of Technology (Sweden) and Zhejiang University (China)

Yasuhiro Koike, Keio University (Japan)

Program Track Chairs

James G. Grote, Photonics Consultant (United States)

Shibin Jiang, AdValue Photonics, Inc. (United States)

Conference Chairs

Hiroshi Fujioka, The University of Tokyo (Japan)

Hadis Morkoç, Virginia Commonwealth University (United States)

Ulrich T. Schwarz, Technische Universität Chemnitz (Germany)

Conference Co-chairs

Jen-Inn Chyi, National Central University (Taiwan)

Jung Han, Yale University (United States)

Motoaki Iwaya, Meijo University (Japan)

Conference Program Committee

Frank Bertram, Otto-von-Guericke-Universität Magdeburg (Germany)

Michal Bockowski, Institute of High Pressure Physics (Poland)

Raffaella Calarco, Paul-Drude-Institut für Festkörperelektronik (Germany)

Mitch M. C. Chou, National Sun Yat-Sen University (Taiwan)

Martin Feneberg, Otto-von-Guericke-Universität Magdeburg (Germany)

Mitsuru Funato, Kyoto University (Japan)

Bernard Gil, Laboratoire Charles Coulomb (France)

Nicolas Grandjean, Ecole Polytechnique Fédérale de Lausanne (Switzerland)

Hideki Hirayama, RIKEN (Japan) 
Ray-Hua Horng, National Chiao Tung University (Taiwan) Chih-Fang Huang, National Tsing Hua University (Taiwan) Michael Kneissl, Technische Universität Berlin (Germany) Elison Matioli, Ecole Polytechnique Fédérale de Lausanne (Switzerland)

Koh Matsumoto, Taiyo Nippon Sanso Corporation (Japan) Hideto Miyake, Mie University (Japan)

Eva Monroy, Commissariat à l'Énergie Atomique (France)

Yong-Tae Moon, LG Electronics Inc. (Korea, Republic of)

Yasushi Nanishi, Ritsumeikan University (Japan)

Ümit Özgür, Virginia Commonwealth University (United States)

Piotr Perlin, Institute of High Pressure Physics (Poland)

Fan Ren, University of Florida (United States)

Tae-Yeon Seong, Korea University (Korea, Republic of)

Bo Shen, Peking University (China)

Jong-In Shim, Hanyang University (Korea, Republic of)

Maria Tchernycheva, Université Paris-Sud 11 (France)

Akio Wakejima, Nagoya Institute of Technology (Japan)

Chih-Chung Yang, National Taiwan University (Taiwan)

Euijoon Yoon, Seoul National University (Korea, Republic of)

Session Chairs

1 Growth I

Ulrich T. Schwarz, Technische Universität Chemnitz (Germany)

2 Growth II

Ferdinand Scholz, Universität Ulm (Germany)

3 Material Characterization I

Frank Bertram, Otto-von-Guericke-Universität Magdeburg (Germany)

4 Material Characterization II

Ulrich T. Schwarz, Technische Universität Chemnitz (Germany)

5 Material Characterization III

Jesús Zúñiga-Pérez, Centre de Recherche sur l'Hétéroepitaxie et ses Applications (France)

$6 \quad$ Doping

Russell D. Dupuis, Georgia Institute of Technology (United States)

7 Nanostructures

Pierre Ruterana, Centre de Recherche sur les lons, les Matériaux et la Photonique (France) 
8 Electron Devices

Hiroshi Fujioka, Institute of Industrial Science, The University of Tokyo (Japan)

9 Laser Diodes

Tetsuya Takeuchi, Meijo University (Japan)

10 VCSEL I

Martin D. Dawson, Fraunhofer UK Research Ltd. (United Kingdom)

11 VCSEL II

Motoaki Iwaya, Meijo University (Japan)

12 Micro LEDs I

Jung Han, Yale University (United States)

13 Micro LEDs II

François Templier, Université Grenoble Alpes, CEA-LETI (France)

14 UV LEDS

Zlatko Sitar, North Carolina State University (United States)

15 LEDs

Vitaliy Avrutin, Virginia Commonwealth University (United States) 
Proc. of SPIE Vol. 10918 1091801-12 Downloaded From: https://www.spiedigitallibrary.org/conference-proceedings-of-spie on 26 Apr 2023
Terms of Use: https://www.spiedigitallibrary.org/terms-of-use 